\title{
Study of Linear Transformation and its Application
}

\author{
Ajay \\ Department of Mathematics \\ Email id: redcross8053@gmail.com \\ Chandigarh University, Mohali, Punjab
}

\begin{abstract}
Linear functions are commonly referred to as "linear map", "linear operator" or "linear transformation". In some cases, the term "homomorphism" will be used, which refers to functions from one kind of set that respect any structures on the sets; The two structures on vector spaces, scalar multiplication and addition, are respected by linear maps from vector spaces. A linear function is commonly written with a capital $\mathrm{L}$ to indicate its linearity, but because we're only looking at very specific functions, we may alternatively express it with merely f. Matrices are a helpful tool for linear transformation calculations. It's important to understand how to find the matrix of a linear transformation as well as the properties of matrices.
\end{abstract}

KEYWORDS: Linear ${ }^{1}$ transformation,rank and nullity theorem, linear differential operators, bases, visualization of linear transformation, application of linear transformation.

\section{INTRODUCTION}

Straight change is one of the more special ideas moved in direct polynomial math. It is similarly one of the ideas with which understudies experience great inconveniences (Sierpinska 2000; Sierpinska et al. 1999)[1-2]. Uicab and Oktaç (2006)[3] saw that a couple of understudies required an express condition for a change related with an issue even in conditions where it isn't needed, as in like manner referred to in Sierpinska (2000)[1].Karrer and Jahn (2008)[4] report various types of difficulties, for instance, change between registers, especially from the reasonable register to others; conviction that direct changes should be applied to polygonal things; and accepting that a change that saves straight lines is basically straight. These makers suggest the usage of a powerful estimation environment where understudies can see a direct change in three registers (sensible, numerical, and system) simultaneously similarly as the effect of carrying out an improvement in one register on the others, to beat these difficulties.

A part of these difficulties may be related to their as of late constructed limit originations, since a straight change is an unprecedented kind of limit between vector spaces. Trigueros and Bianchini (2016)[7] saw that concerning a showing issue this relationship turns out to be all the more clear for understudies. In our examination gathering, we have been thinking about the straight change idea from different focuses, including how it is fabricated, related difficulties, originations that 
understudies may make, and depictions. In this part the point is to bring to the thought of a worldwide group picked work that has been driven in Spanish about the learning of this idea. The revelations uncovered here structure part of a greater consistent endeavor about the perception of Linear Algebra ideas. In hate of the way that the data uncovered comes from inspects drove in Mexico and Chile, the saw wonders may uncover knowledge into challenges that understudies understanding in various bits of the world as well.Mathematically, we noticed the very sorts of conceptions and instinctive models that understudies had created as in the past examination. Arithmetically, however, this was not the situation. Mathematical images, instead of pictures of mathematical items, are not "offered straightforwardly to the brain" (Sierpinska 2000, p. 233)[5]; the impact of the transformation on the vectors isn't promptly accessible in the tuple documentation. ${ }^{5}$ In this specific situation, algorithmic reasoning overwhelmed. Encounter of various responses to the equal issues in mathematical and arithmetical settings assisted understudies with perceiving their errors, yet we don't know how much it was useful in building the concept.

Our first assessment toward this way (Molina and Oktaç 2007)[6] put highlight on numerical settings, since these are upheld less in direct polynomial Mathematical courses. Undoubtedly, in the wake of examining some straight polynomial numerical understanding materialFor instance, Trigueros and Bianchini (2016)[7] saw that understudies had a more troublesome time finding the recipe of a turn (since it contains mathematical capacities) when chipping away at a displaying issue than with the equation of a shear transformation; in this setting revolution is viewed as more intricate.As Hillel $(2000)$ [8, 9, 10, 11] remarks, not all linear transformations have straightforward mathematical understandings, even in two and three dimensional spaces. Nonetheless, for understudies these models that are related with specific developments in the plane supplant the definition, offering ascend to instinctive models that fill in for numerical hypothesis (Fischbein 1989) [12].

These models force certain "results" in the substitute hypothesis; for instance, "a linear transformation does likewise to all vectors." Since this assertion is a rearranged form of what befalls the plane mathematically under a linear transformation, "doing likewise" is additionally deciphered in an oversimplified way outwardly. As per Fischbein (1989) [12] "the instinctive model controls from in the background, the importance, the utilization, the properties of the officially settled concept. The natural model is by all accounts more grounded than the conventional concept".

\section{Binary operation:}

A calculation that combines two items to create a new one is known as binary operation. A binary operation is a two-arity operation that requires the use of dual sets of numbers. For example, scalar multiplication of vector spaces produces a vector by taking a scalar and a vector, and scalar product produces a scalar by taking two vectors. 
Instead of using functional notation of the form $\mathrm{f}$, binary operations are represented as $a+b, a * b$, a.b, or ab $(a, b)$. Without the operator, powers are written with the second argument superscripted.

The set of triples $(\mathrm{a}, \mathrm{b}, \mathrm{f}(\mathrm{a}, \mathrm{b})$ ) in $\mathrm{S} \times \mathrm{S} \times \mathrm{S}$ for any a and $\mathrm{b}$ in $\mathrm{S}$ given a binary operation $\mathrm{f}$ on a set $\mathrm{S}$ is called a ternary relation on $\mathrm{S}$.

\section{Linear Transformations:}

A linear transformation is a transformation. $F: R_{n} \rightarrow R_{m}$ satisfying the following properties.

$\mathrm{F}(\mathrm{u}+\mathrm{v})=\mathrm{F}(\mathrm{u})+\mathrm{F}(\mathrm{v})$

$\mathrm{F}(\mathrm{cu})=\mathrm{cF}(\mathrm{u})$

for all vectors $u, v \in R_{n}$.

Let $F: R_{n} \rightarrow R_{m}$ be a matrix transformation: $F(x)=A x$ for an $m \times n$ matrix $A$. By this proposition, we have

$\mathrm{F}(\mathrm{u}+\mathrm{v})=\mathrm{A}(\mathrm{u}+\mathrm{v})$

$=\mathrm{Au}+\mathrm{Av}$

$=\mathrm{F}(\mathrm{u})+\mathrm{F}(\mathrm{v})$

$\mathrm{F}(\mathrm{cu})=\mathrm{A}(\mathrm{cu})$

$=\mathrm{cAu}$

$=\mathrm{CF}(\mathrm{u})$

for all vectors $\mathrm{u}, \mathrm{v}$ in $\mathrm{R}_{\mathrm{n}}$. A linear transformation is one that satisfies the two defining properties of a matrix transformation.

The opposite is valid, as we'll see in the next section: any linear transformation is a matrix transformation; we just haven't computed its matrix yet.

Let $\mathrm{T}: \mathrm{R}_{\mathrm{n}} \rightarrow \mathrm{R}_{\mathrm{m}}$ be a linear transformation.

1. $\mathrm{T}(0)=0$.

2. For any vectors $\mathrm{v}_{1}, \mathrm{v}_{2}, \ldots, \mathrm{v}_{\mathrm{k}}$ in $\mathrm{R}_{\mathrm{n}}$ and scalars $\mathrm{c}_{1}, \mathrm{C}_{2}, \ldots, \mathrm{C}_{\mathrm{k}}$, we have

$$
\mathrm{T}\left(\mathrm{c}_{1} \mathrm{v}_{1}+\mathrm{c}_{2} \mathrm{v}_{2}+\cdots+\mathrm{c}_{\mathrm{k}} \mathrm{V}_{\mathrm{k}}\right)=\mathrm{c}_{1} \mathrm{~T}\left(\mathrm{v}_{1}\right)+\mathrm{c}_{2} \mathrm{~T}\left(\mathrm{v}_{2}\right)+\cdots+\mathrm{c}_{\mathrm{k}} \mathrm{T} \mathrm{s}\left(\mathrm{v}_{\mathrm{k}}\right) \text {. }
$$

This is why linear functions are so attractive; they are, in fact, relatively simple functions due to two characteristics:

1. They act on vector spaces.

2. They act additively and homogenously. 
A linear transformation with domain $\mathrm{R}^{3}$ is completely specified by the way it acts on three vectors

$\left(\begin{array}{l}0 \\ 1 \\ 0\end{array}\right),\left(\begin{array}{l}1 \\ 0 \\ 0\end{array}\right),\left(\begin{array}{l}0 \\ 0 \\ 1\end{array}\right)$

Similarly, the action of a linear transformation with domain $\mathrm{R}^{\mathrm{n}}$ on the $\mathrm{n}$ distinct $\mathrm{n}$-vectors with exactly one non-zero component completely specifies the transformation, and the matrix from may be retrieved from this information.

\section{Linear Differential Operators}

When we stopped using the limit description of the derivative, studied the power law, and began using the linearity of the derivative operator in calculus classes, things got a lot simpler.

Eg:- let $\mathrm{T}$ be the vector space of degree 2 or less with standard addition and scalar multiplication polynomials;

$$
\mathrm{T}=\left\{\mathrm{b}_{0} .1+\mathrm{b}_{1} \mathrm{y}+\mathrm{b}_{2} \mathrm{y}^{2} \mid \mathrm{b}_{0}, \mathrm{~b}_{1}, \mathrm{~b}_{2} \in \mathrm{R}\right\}
$$

Let $\frac{d}{d y}: \mathrm{T} \rightarrow \mathrm{T}$ be the derivative operator. The equations, along with linearity of the derivative operator, allow one to take the derivative of any $2^{\text {nd }}$ degree polynomial:

$\frac{d}{d y} 1=0, \frac{d}{d y} y=1, \frac{d}{d y} y^{2}=2 y$.

In particular

$$
\frac{d}{d y}\left(b_{0} 1+b_{1} y+b_{2} y^{2}\right)=b_{0} \frac{d}{d y} 1+b_{1} \frac{d}{d y} y+b_{2} \frac{d}{d y} y^{2}=0+b_{0}+2 b_{2} y .
$$

As a result, the derivative of any of the infinitely many second order polynomials is determined by only three inputs.

\section{Bases $^{1}$}

The function of linear algebra is to take use of linear functions' latent clarity. It seems out that there is a lot of choice in how to go about doing this. It is because of this freedom that linear algebra is so strong.

We see that a linear operator acting on $\mathrm{R}^{2}$ is completely specified by how it acts on the pair of vectors $\left(\begin{array}{l}1 \\ 0\end{array}\right)$ and $\left(\begin{array}{l}0 \\ 1\end{array}\right)$. In fact, any linear operator acting on $\mathrm{R}^{2}$ is also completely specified by how it acts on the pair of vectors $\left(\begin{array}{l}1 \\ 1\end{array}\right)$ and $\left(\begin{array}{c}1 \\ -1\end{array}\right)$.

Eg:- the linear operator $\mathrm{L}$ is a linear operator then it is completely specified by two equalities

$L\left(\begin{array}{l}1 \\ 1\end{array}\right)=\left(\begin{array}{l}3 \\ 6\end{array}\right)$ and $s L\left(\begin{array}{c}1 \\ -1\end{array}\right)=\left(\begin{array}{l}5 \\ 4\end{array}\right)$. 
This is because any vector $\left(\begin{array}{l}x \\ y\end{array}\right)$ in $\mathrm{R}^{2}$ is a sum of multiples of $\left(\begin{array}{l}1 \\ 1\end{array}\right)$ and $\left(\begin{array}{c}1 \\ -1\end{array}\right)$ which can be calculated via a linear systems problems as follows:

$$
\begin{gathered}
\left(\begin{array}{l}
x \\
y
\end{array}\right)=a\left(\begin{array}{l}
1 \\
1
\end{array}\right)+b\left(\begin{array}{c}
1 \\
-1
\end{array}\right) \\
\left(\begin{array}{cc}
1 & 1 \\
1 & -1
\end{array}\right)\left(\begin{array}{l}
a \\
b
\end{array}\right)=\left(\begin{array}{l}
x \\
y
\end{array}\right) \\
\left(\begin{array}{cc|c}
1 & 1 & x \\
1 & -1 & y
\end{array}\right) \sim\left(\begin{array}{ll|l}
1 & 0 \\
0 & 1 & \frac{x+y}{2} \\
2
\end{array}\right) \\
\left\{\begin{array}{l}
a=\frac{x+y}{2} \\
b=\frac{x-y}{2}
\end{array}\right.
\end{gathered}
$$

Thus

$$
\left(\begin{array}{l}
x \\
y
\end{array}\right)=\frac{x+y}{2}\left(\begin{array}{l}
1 \\
1
\end{array}\right)+\frac{x-y}{2}\left(\begin{array}{c}
1 \\
-1
\end{array}\right)
$$

Then, by first expressing the vector as a sum of multiples and then applying linearity, we can calculate how $\mathrm{L}$ behaves on any vector;

$$
\begin{gathered}
L\left(\begin{array}{l}
x \\
y
\end{array}\right)=L\left[\frac{x+y}{2}\left(\begin{array}{l}
1 \\
1
\end{array}\right)+\frac{x-y}{2}\left(\begin{array}{c}
1 \\
-1
\end{array}\right)\right] \\
=\frac{x+y}{2} L\left(\begin{array}{l}
1 \\
1
\end{array}\right)+\frac{x-y}{2} L\left(\begin{array}{c}
1 \\
-1
\end{array}\right) \\
=\frac{x+y}{2}\left(\begin{array}{l}
3 \\
6
\end{array}\right)+\frac{x-y}{2}\left(\begin{array}{c}
5 \\
4
\end{array}\right) \\
=\left(\begin{array}{l}
\frac{3 x+3 y}{2} \\
3 x+3 y
\end{array}\right)+\left(\begin{array}{l}
\frac{5 x-5 y}{2} \\
2 x-2 y
\end{array}\right) \\
=\left(\begin{array}{l}
4 x-y \\
5 x-y
\end{array}\right)
\end{gathered}
$$

As a result, the value of $L$ at just two inputs absolutely defines it.

It should not surprise to learn there are infinitely many pairs of vectors from $\mathrm{R}^{2}$ with the property that any vector can be expressed as a linear combination of them any pair that when used as columns of a matrix give an invertible matrix works. Such a pair is known as a basis for $\mathrm{R}^{2}$.

Similarly, there are infinitely many triples of vectors with the property that any vector from $\mathrm{R}^{3}$ is expressed as a linear combination of them these are the triples that accustomed columns of a matrix give an invertible matrix. Such a triple is named a basis for $\mathrm{R}^{3}$. 
During a similar spirit, there are infinitely many pairs of vectors with the property that each vector in

$$
T=\left\{c_{1}\left(\begin{array}{l}
0 \\
1 \\
1
\end{array}\right)+c_{2}\left(\begin{array}{l}
1 \\
1 \\
0
\end{array}\right) \mid c_{1}, c_{2} \in R\right\}
$$

Is expressed as a linear combination of them. Some examples are

$$
T=\left\{c_{1}\left(\begin{array}{l}
0 \\
1 \\
1
\end{array}\right)+c_{2}\left(\begin{array}{l}
2 \\
2 \\
0
\end{array}\right) \mid c_{1}, c_{2} \in R\right\}=\left\{c_{1}\left(\begin{array}{l}
1 \\
5 \\
3
\end{array}\right)+c_{2}\left(\begin{array}{l}
0 \\
1 \\
1
\end{array}\right) \mid c_{1}, c_{2} \in R\right\}
$$

Such a pair could be a called a basis for V.

You most likely have some intuitive notion of what dimension means. Roughly speaking, dimension is that the number of independent directions available. To work out the dimension of a vector space, I stand at the origin, and pick a direction. If there are any vectors in my vector space that aren't there in direction, then I select another direction that isn't within the line determined by the direction I chose. If there are any vectors in my vector space not within the plane determined by the primary two directions, then I select one inevery of them as my next direction. A minimal set of independent vectors is named a basis. The quantity of vectors in my basis is that the dimension of the vector space. Every vector space has many bases, but all bases for a selected vector space have the identical number of vectors. Thus dimension may be a welldefined concept.

The fact that each vector space (over R) has infinitely many bases is really very useful. Often a decent choice of basis can reduce the time required to run a calculation in dramatic ways!

A basis could be a set of vectors in terms of which it'spossible to uniquely express the other vector.

\section{Rank-nullity theorem:-}

A linear transformation may be a special kind of function from one vector space to a different. Once we later specialize to linear transformation, we'll also find some ways to making subspaces.

It is a theorem in algebra, which asserts that the dimension of the domain of a linear map is that the sum of its rank (the dimension of its image) and its nullity (the dimension of its kernel)

let V,U be a linear vector spaces, where V may be a finite dimensional .

$\operatorname{rank}(\mathrm{T})+\operatorname{Nullity}(\mathrm{T})=\operatorname{Dim}(\mathrm{V})$

here $\mathrm{T}$ is that the transformation from $\mathrm{V}$ to $\mathrm{U}$. also here $\operatorname{rank}(\mathrm{T})=\operatorname{dim}(\operatorname{image}(\mathrm{T}))$

and nullity $(\mathrm{T})=\operatorname{dim}(\operatorname{ker}(\mathrm{T}))$. 
Let $\mathrm{V}$ and $\mathrm{W}$ be a vector space over $\mathrm{F}$ and let $\mathrm{T} \epsilon(\mathrm{V}, \mathrm{W})$. if $\operatorname{dim}(\mathrm{V})=\operatorname{dim}(\mathrm{W})$ then, the subsequent are equivalent.

1) $\mathrm{T}$ is one-one.

2) $\operatorname{Ker}(\mathrm{T})=\{0\}$

3) $\mathrm{T}$ is onto.

4) $\operatorname{Dim}(\operatorname{Rng}(T))=\operatorname{dim}(V)$.

\section{Visualizing linear transformation}

a. Algebra of linear transformation[Sum and scalar multiplication of linear transformation]

The idea of a transformation can seem more complicated than it really is at first, so before diving into how $2 \times 2$ matrices transform two-dimensional space, or how $3 \times 3$ matrices transform three dimension space.

Let $\mathrm{V}, \mathrm{W}$ be a vector spaces over $\mathrm{F}$ and let $\mathrm{S}, \mathrm{T} \in(\mathrm{V}, \mathrm{W})$. Then we define the point-wise

Sum of $S$ and $T$, denoted $S+T$, by $(S+T)(v)=S(v)+T(v)$, for all $v \in V$.

Scalar multiplication, denoted cT for c $\epsilon$ F, by $(c T)(v)=c(T(v))$, for all $v \in V$.

b. Equality of linear transformation:- Let $S, T \in(V, W)$. Then, $S$ and $T$ are said to be equal if $S(x)=T(x)$. for all $x \epsilon V$.

c. Singular and non-singular transformation:-

Let $U$ and $V$ be a vector spaces over $F$ and let $T \epsilon(U, V)$. Then $T$ is said to be singular if $0 \subsetneq \operatorname{Ker}(\mathrm{T})$. That is $\operatorname{ker}(\mathrm{T})$ is not a zero vector. If $\operatorname{Ker}(\mathrm{T})$ is non zero then, $\mathrm{T}$ is called non-singular.

\section{d. Isomorphism of vector space:-}

Let $U$ and $V$ be two vector spaces over $F$ and let $T \epsilon(U, V)$, then $T$ is said to be an isomorphism if $\mathrm{T}$ is bijective. The vector spaces $\mathrm{V}$ and $\mathrm{U}$ are said to be isomorphic, denoted $\mathrm{U} \cong \mathrm{V}$, if there is an isomorphism from $\mathrm{U}$ to $\mathrm{V}$.

\section{Application of Linear Transformation}

1. The geometry of linear transformation in the plane

2. Reflection in $x$-axis, $y$-axis, and $y=x$

3. Horizontal and vertical expansion and contraction

4. Horizontal and vertical shear

5. Computer graphics(to produce any desire angle of view of a 3-D figure ) 


\section{ACKNOWLEDGMENTS}

I will be very much thankful to my professor, Dr. Sangeeta kumari to help me for motivation on research field.

\section{CONCLUSION}

In this paper we examined about representation and comprehension of direct change. There were different suspicions and bits of hearsay identified with the idea of direct change which are clashed in this paper prompting a superior agreement and information about straight change .A linear transformation obtain inverse if and only if it's bijective (one-one and onto). We've also seen that linear transformations are frequently represented by matrices, and we've seen a number of methods for determining if a matrix is invertible.

\section{REFERENCES}

[1]Sierpinska, Anna. "On some aspects of students' thinking in linear algebra." On the teaching of linear algebra. Springer, Dordrecht, 2000. 209-246

[2] Sierpinska, A., Dreyfus, T., \& Hillel, J. (1999). Evaluation of a teaching design in linear algebra: The case of linear transformations. Recherches en didactique des mathématiques (Revue), 19(1), 7-40.

[3] Uicab, R., \& Oktaç, A. (2006). Transformaciones lineales en un ambiente de geometría dinámica: proyecto CONACYT 2002-C01-41726S. RELIME. Revista latinoamericana de investigación en matemática educativa, 9(3), 459-490.

[4] Registrant WHOIS contact information verification | Namecheap.com. Tsg.icme11.org. (2021). Retrieved 12 July 2021, from http://tsg.icme11.org/document/get/237.

[5] Sierpinska, A. (2000). On some aspects of students' thinking in linear algebra. In On the teaching of linear algebra (pp. 209-246). Springer, Dordrecht.

[6] Molina, J. G., \& Oktaç, A. (2007). Concepciones de la transformación lineal en contexto geométrico. Revista latinoamericana de investigación en matemática educativa, 10(2), 241-273.

[7]Trigueros, M., \& Bianchini, B. (2016). Learning linear transformations using models. First Conference of International Network for Didactic Research in University Mathematics. Montpellier,France. https://hal.archives-ouvertes.fr/hal-01337884/document

[8]Sierpinska, A., Dreyfus, T., \& Hillel, J. (1999). Evaluation of a teaching design in linear algebra: The case of linear transformations. Recherches en didactique des mathématiques (Revue), 19(1), 7-40.

[9] Dorier, J. (2000). On the teaching of linear algebra (pp. 191-207). Kluwer Academic Publishers. 
[10]Dreyfus, T., Hillel, J., \& Sierpinska, A. (1998). Cabri-based linear algebra: transformations. In Proceedings of CERME-1 (First Conference on European Research in Mathematics Education), Osnabrück.

[11]Fischbein, E. (1989). Tacit models and mathematical reasoning. For the learning of mathematics, 9(2), 9-14.

[12]I. B. Wilkinson, S. A. Fuchs, I. M. Jansen et al., "Reproducibility of pulse wave velocity and augmentation index measured by pulse wave analysis," Journal of Hypertension, vol. 16, no. 12, pp. 2079-2084, 1998.

[13]J. Blacher, R. Asmar, S. Djane, G. M. London, and M. E. Safar, "Aortic pulse wave velocity as a marker of cardiovascular risk in hypertensive patients," Hypertension, vol. 33, no. 5, pp. 1111-1117, 1999.

[14]J. Blacher, A. P. Guerin, B. Pannier, S. J. Marchais, M. E. Safar, and G. M. London, "Impact of aortic stiffness on survival in end-stage renal disease," Circulation, vol. 99, no. 18, pp. 2434-2439, 1999.

[15]K. Cruickshank, L. Riste, S. G. Anderson, J. S. Wright, G. Dunn, and R. G. Gosling, "Aortic pulse-wave velocity and its relationship to mortality in diabetes and glucose intolerance: an integrated index of vascular function?" Circulation, vol. 106, no. 16, pp. 2085-2090, 2002. 